\title{
Effect of strongly coupled plasma on the spectra of hydrogenlike carbon, aluminium and argon
}

\author{
S. Bhattacharyya ${ }^{1}$, A.N. Sil ${ }^{2,3, a}$, S. Fritzsche ${ }^{4}$, and P.K. Mukherjee ${ }^{3}$ \\ 1 Kandi Raj College, Kandi, Murshidabad, West Bengal 742 137, India \\ 2 Max Planck Institute for the Physics of Complex Systems, Nöthnitzer Str. 38, 01187 Dresden, Germany \\ 3 Indian Association for the Cultivation of Science, Jadavpur, Kolkata-700 032, India \\ 4 Universität Kassel, Institut für Physik, 34109 Kassel, Germany
}

Received 12 April 2007 / Received in final form 24 July 2007

Published online 21 September 2007 - (C) EDP Sciences, Società Italiana di Fisica, Springer-Verlag 2007

\begin{abstract}
A detailed study has been performed for estimating the orbital energies, positions and shifts of the Lyman lines of $\mathrm{C}^{5+}, \mathrm{Al}^{12+}$ and $\mathrm{Ar}^{17+}$ under strongly coupled plasma with a view to understand such line positions and shifts obtained in laser produced plasma experiments. The effect of strongly coupled plasma has been treated within the Ion Sphere (IS) model. Both non-relativistic and relativistic methods have been used for estimating the spectral properties. Theoretical estimates with IS model of the plasma are in conformity with the results of laser plasma experiments on these highly stripped ions. The experimental data for the systems have also been compared with the theoretical estimates using Debye screening model of the plasma with spatial confinements which gives additional restrictions to the wave functions at finite boundaries.
\end{abstract}

PACS. 95.30.Qd Magnetohydrodynamics and plasmas - 52.27.Gr Strongly-coupled plasmas 52.70.-m Plasma diagnostic techniques and instrumentation - 52.72. $+\mathrm{v}$ Laboratory studies of space- and astrophysical-plasma processes

\section{Introduction}

The spectral properties of atomic systems are modified considerably under external confinements [1-3]. Of particular interest, is the effect of a surrounding plasma of different coupling strengths $\Gamma$, defined as the ratio of average Coulomb potential energy between pairs of particles and their kinetic energy. For weakly coupled plasma $\Gamma<1$ and one can apply the standard Debye screening model [4] in which the potential energy between charged particles is represented by a screened Coulomb potential. The condition $\Gamma \geq 1$ refers to strongly coupled plasma in which the potential energy function, though simple, is of completely different nature than in a Debye screening model [5]. Such plasma conditions prevail in, highly evolved stars, the interior of Jovian planets, explosive shock tubes, two dimensional states of electrons trapped in surface states of liquid helium, laser produced and inertial confinement fusion plasmas $[5,6]$. Recent experimental observations using laser produced plasmas [7-12] open up an interesting field for the theoretical investigations along this line. Such high density plasmas are of particular interest in astrophysics and inertial confinement fusion processes. The X-ray opacity of matter under stellar interior conditions and the X-ray diagnostics of ICF plasmas can

\footnotetext{
${ }^{\mathrm{a}}$ e-mail: amar@mpipks-dresden.mpg.de
}

be achieved from such a study [11]. Effect of dense plasma on the ionization potential, collision and photo absorption cross sections, fine structure splitting and spectral line shifts have been investigated earlier by Stewart and Pyatt [13], Rozsnai [14], Ray [15], Jung [16], Griem [17], Siedel et al. [18] and Skupski [19]. Applications of density functional approach along this line was reviewed by Gupta and Rajagopal [20].

In the current context, we will focus our attention to the experimental findings based on time and space resolved extreme ultraviolet spectra of carbon plasmas with 100 fs laser pulses [10], inertially confined laser imploded Ar plasma [11] and ultrashort laser produced $\mathrm{Al}$ plasma [12]. For such laser produced plasmas $\Gamma>1$ and one can apply strongly coupled plasma model to investigate the spectral properties of isoelectronic ions of hydrogen. In this communication we would like to investigate in detail the effect of strongly coupled plasma on the Lyman lines of highly stripped carbon, aluminium and argon. Ion Sphere (IS) model of the plasma [5] has been utilized for such a study. Our motivation is to investigate how the simple IS model is effective in obtaining results which can be compared favourably with the experimentally observed values. In addition we would also like to investigate the applicability of the Debye plasma model with a spherical confinement on the spectral line positions and shifts of the Lyman lines under the laser plasma experimental 
conditions [10-12] and to estimate the shifts in ionization potentials. Such studies have been done earlier for hydrogen $[21,22]$ and helium like systems $[23,24]$ to understand the behavior of the structural properties of one and two electron systems under weak as well as strongly coupled plasmas. A brief outline of the theory is given in Section 2 and a discussion of the results follow in Section 3.

\section{Theory}

In presence of an external plasma environment the potential energy is modified and the non-relativistic Hamiltonian of a hydrogen like atomic system [a.u. is used throughout] can be represented by

$$
H_{0}=-\frac{1}{2} \nabla^{2}+V_{\text {eff }}(r)
$$

where the structure of the one body effective potential depends on the type of the coupling of the plasma with the atomic charge cloud. For the relativistic treatment appropriate modification of the kinetic energy part of the Hamiltonian is done through the introduction of the Dirac operators. Once the form of the Hamiltonian is known the effect of the external plasma can be taken care of by using a suitable form of the effective one particle potential. Currently we are interested in the case of strongly coupled plasma for which $\Gamma \geq 1$. For such a case the model which is usually referred to as the Ion Sphere (IS) model [5] has been adopted here for the estimation of line shifts. Physically the IS model assumes a smeared out uniform electron density distribution within a sphere and zero density outside $[5,13]$. In this model for the case of a homogeneous one component plasma surrounding an ion of nuclear charge $Z$ having one valence electron like hydrogenic ions described here, one can define a sphere of radius $R$ (usually referred to as the Wigner-Seitz radius) such that the plasma electrons contained in this sphere together with the valance electron completely neutralize the central positive charge; thus maintaining the overall charge neutrality of the system within this sphere $[5,13,20]$. Since the total charge within the Wigner-Seitz sphere is important one can use a volume averaged density for performing the calculation as existing in literature $[13,14]$. In such a situation the Wigner-Seitz radius $R$ can be represented by

$$
R=\left[\frac{(Z-1)}{\frac{4}{3} \pi n}\right]^{\frac{1}{3}}
$$

where $n$ is the average electron density within the sphere.

The expression for the potential can easily be obtained from classical electrostatics as

$$
V_{\text {eff }}(r)=-\frac{Z}{r}+\frac{(Z-1)}{2 R}\left[3-\left(\frac{r}{R}\right)^{2}\right] .
$$

In order to analyze the energy of the system for different coupling strengths of the plasma reflected in $R$, one has to solve the appropriate Schrödinger equation

$$
H_{0} \psi=E_{0} \psi
$$

subject to the normalization constant

$$
\langle\psi \mid \psi\rangle=1
$$

For the relativistic case the corresponding Dirac equation is to be solved. It is assumed that no electron current takes place at the boundary surface defined by the WignerSeitz radius $R$ and the wave function is assumed to satisfy the boundary condition Stewart [13], Rozsnai [14], and Skupski [19]

$$
\psi(r)=0 \quad \text { at } \quad r=R .
$$

Other boundary conditions involving vanishing of the first derivative of the orbital at the boundary can also be adopted as was done by Rozsnai [14]. However, we have taken the boundary condition given by equation (6) for computational simplicity. Such conditions can always be satisfied by choosing the basis sets appropriately. We represent the radial part of the orbital

$$
\psi(r)=(R-r) \chi(r)
$$

where $\chi(r)$ is a linear combination of Slater type orbitals (STO)

$$
\chi(r)=\sum_{i} C_{i} r^{n_{i}} e^{-\rho_{i} r} .
$$

The analytical solution of hydrogen like problem in a plasma is difficult. We adopt the basis set expansion technique for obtaining the energy of the ground state in a plasma environment for the non relativistic calculation. The non linear parameters $n_{i}$ and $\rho_{i}$ for the ground orbital are preassigned from physical considerations and the linear coefficients are determined from the solution of the generalized eigenvalue equation

$$
\mathbf{H}_{0} \mathbf{C}=E_{0} \mathbf{S} \mathbf{C}
$$

where $\mathbf{H}_{0}$ and $\mathbf{S}$ are the Hamiltonian and the overlap matrices with respect to the basis sets and $\mathbf{C}$ is the coefficient vector. Solution of equation (9) yields the ground state energy and the linear coefficients at different plasma coupling strengths which are functions of the plasma parameters. All the integrals are to be evaluated at finite domain radius $R$. The appropriate integral package has been developed. For the relativistic case a numerical evaluation of the energies is sought using Dirac Hamiltonian and the standard relativistic program package RATIP as developed by Fritzsche et al. [25].

In addition to evaluation of the ground state energies at different plasma coupling strengths we apply an external time dependent perturbation [21-24] for probing the low lying excited states of the system. The transition energies and excited state wave functions have been calculated using linear response theory under such a perturbation [21-24]. The procedure yields the spectral line positions and other excitation properties under the plasma environment. 
Table 1. Relativistic and non-relativistic transition energy of $\mathrm{Al}^{12+}$ for different Ion-Sphere (IS) radius.

\begin{tabular}{|c|c|c|c|c|c|c|c|c|}
\hline \multirow{2}{*}{$\begin{array}{l}\text { Ion-sphere } \\
\text { radius (a.u.) }\end{array}$} & \multirow{2}{*}{$\begin{array}{c}\text { Plasma } \\
\text { density } n_{e} / \text { c.c. }\end{array}$} & \multicolumn{2}{|c|}{ Orb Ener (a.u.) } & \multirow{2}{*}{$\begin{array}{r}\text { Transition } \\
\text { scheme }\end{array}$} & \multicolumn{2}{|c|}{ Transition energy (a.u.) } & \multicolumn{2}{|c|}{ Energy shift (eV) } \\
\hline & & Rel & Non-Rel & & Rel & Non-Rel & Rel & Non-Rel \\
\hline \multirow[t]{3}{*}{$\infty$} & & 84.69 & 84.50 & $1 s \rightarrow 2 p$ & 63.53747 & 63.37500 & & \\
\hline & & & & $\rightarrow 3 p$ & 75.28958 & 75.11111 & & \\
\hline & & & & $\rightarrow 4 p$ & 79.40327 & 79.21875 & & \\
\hline \multirow[t]{3}{*}{9.9} & $1.99(+22)$ & 82.8729 & 82.6819 & $1 s \rightarrow 2 p$ & 63.53715 & 63.37401 & 0.0087 & 0.0269 \\
\hline & & & & $\rightarrow 3 p$ & 75.28855 & 75.10482 & 0.0280 & 0.1709 \\
\hline & & & & $\rightarrow 4 p$ & 79.38251 & 79.19697 & 0.5649 & 0.5927 \\
\hline \multirow[t]{3}{*}{5.7822} & $1.0(+23)$ & 81.5785 & 81.3876 & $1 s \rightarrow 2 p$ & 63.53319 & 63.37003 & 0.1165 & 0.1352 \\
\hline & & & & $\rightarrow 3 p$ & 75.26206 & 75.09233 & 0.7489 & 0.5108 \\
\hline & & & & $\rightarrow 4 p$ & 79.29516 & 79.10701 & 2.9418 & 3.0406 \\
\hline \multirow[t]{3}{*}{3.38146} & $5.0(+23)$ & 79.3706 & 79.1796 & $1 s \rightarrow 2 p$ & 63.51337 & 63.35014 & 0.6558 & 0.6765 \\
\hline & & & & $\rightarrow 3 p$ & 75.12798 & 74.98010 & 4.3974 & 3.5647 \\
\hline & & & & $\rightarrow 4 p$ & 78.86261 & 78.70955 & 14.7121 & 13.8540 \\
\hline \multirow[t]{3}{*}{3.18207} & $6.0(+23)$ & 79.0376 & 78.8467 & $1 s \rightarrow 2 p$ & 63.50841 & 63.34516 & 0.7908 & 0.8120 \\
\hline & & & & $\rightarrow 3 p$ & 75.09388 & 74.94203 & 5.3253 & 4.6006 \\
\hline & & & & $\rightarrow 4 p$ & 78.75720 & 78.66120 & 17.5805 & 15.1717 \\
\hline \multirow[t]{2}{*}{3.0227} & $7.0(+23)$ & 78.7399 & 78.5489 & $1 s \rightarrow 2 p$ & 63.50344 & 63.34018 & 0.9260 & 0.9475 \\
\hline & & & & $\rightarrow 3 p$ & 75.05951 & 74.90371 & 6.2605 & 5.6434 \\
\hline \multirow[t]{2}{*}{2.89111} & $8.0(+23)$ & 78.4694 & 78.2784 & $1 s \rightarrow 2 p$ & 63.49847 & 63.33519 & 1.0612 & 1.0833 \\
\hline & & & & $\rightarrow 3 p$ & 75.02489 & 74.86516 & 7.2026 & 6.6924 \\
\hline \multirow[t]{2}{*}{2.7798} & $9.0(+23)$ & 78.2206 & 78.0297 & $1 s \rightarrow 2 p$ & 63.49349 & 63.33019 & 1.1968 & 1.2193 \\
\hline & & & & $\rightarrow 3 p$ & 74.98993 & 74.82618 & 8.1539 & 7.7531 \\
\hline \multirow[t]{2}{*}{2.68386} & $1.0(+24)$ & 77.9897 & 77.7987 & $1 s \rightarrow 2 p$ & 63.48851 & 63.32519 & 1.3323 & 1.3554 \\
\hline & & & & $\rightarrow 3 p$ & 74.95463 & 74.78648 & 9.1145 & 8.8334 \\
\hline \multirow[t]{2}{*}{2.13018} & $2.0(+24)$ & 76.2519 & 76.0610 & $1 s \rightarrow 2 p$ & 63.43847 & 63.27495 & 2.6939 & 2.7225 \\
\hline & & & & $\rightarrow 3 p$ & 74.58295 & 74.31376 & 19.2284 & 21.6967 \\
\hline \multirow[t]{2}{*}{1.97749} & $2.5(+24)$ & 75.6022 & 75.4113 & $1 s \rightarrow 2 p$ & 63.41328 & 63.24965 & 3.3794 & 3.4109 \\
\hline & & & & $\rightarrow 3 p$ & 74.38536 & 74.03433 & 24.6051 & 29.3004 \\
\hline \multirow[t]{2}{*}{1.86089} & $3.0(+24)$ & 75.0346 & 74.8437 & $1 s \rightarrow 2 p$ & 63.38798 & 63.22420 & 4.0678 & 4.1035 \\
\hline & & & & $\rightarrow 3 p$ & 74.18693 & 73.75165 & 30.0046 & 36.9925 \\
\hline \multirow[t]{2}{*}{1.76768} & $3.5(+24)$ & 74.5273 & 74.3364 & $1 s \rightarrow 2 p$ & 63.36256 & 63.19856 & 4.7595 & 4.8012 \\
\hline & & & & $\rightarrow 3 p$ & 73.98516 & 73.47956 & 35.4951 & 44.3965 \\
\hline 1.4770 & $6.0(24)$ & 72.5370 & 72.3461 & $1 s \rightarrow 2 p$ & 63.23364 & 63.06557 & 8.2676 & 8.4800 \\
\hline 1.3419 & $8.0(24)$ & 71.3210 & 71.1306 & $1 s \rightarrow 2 p$ & 63.12816 & 62.94926 & 11.1379 & 11.5850 \\
\hline 1.2457 & $1.0(25)$ & 70.2961 & 70.1059 & $1 s \rightarrow 2 p$ & 63.02080 & 62.82014 & 14.0593 & 15.0985 \\
\hline
\end{tabular}

To be more specific we apply a harmonic perturbation on the system

$$
H^{\prime}(\mathbf{r}, t)=g(\mathbf{r}) e^{-i \omega t}+g^{\dagger}(\mathbf{r}) e^{i \omega t}
$$

where $g(\mathbf{r})$ is an one particle perturbation operator. The angular part of the perturbation operator is so chosen as to get a dipolar excitation from the ground state enabling us to compare the laser spectroscopic data. The external perturbation changes the ground state wave function $\psi$ to the perturbed function $\phi$. The first order perturbation correction to the ground state wave function oscillates harmonically and its spatial part can be evaluated through the optimization of a standard variational functional [26]

$$
J(\phi)=\frac{1}{T} \int_{0}^{T} d t \frac{\left\langle\phi\left|H_{0}+H^{\prime}-i \frac{\partial}{\partial t}\right| \phi\right\rangle}{\langle\phi \mid \phi\rangle}
$$

with

$$
\delta J(\phi)=0 .
$$

The optimization is carried out with respect to linear variation parameters introduced in the first order correction to the ground state function $\psi$. The procedural details have been given elsewhere [21-24]. The basis sets for the perturbed functions are similar to that given by equations (7) and (8) with different linear and non linear parameters. The functional has poles at certain frequency $\omega$, the positions of which indicate the singly excited states of the system. One can extract the transition properties from a study of the pole positions [21]. For the relativistic case RATIP package generates the relevant excitation properties directly [25]. A discussion of the results is given in the next section.

\section{Results and discussions}

The effect of strongly coupled plasma on the orbital energy and low lying excited states of $\mathrm{C}^{5+}, \mathrm{Al}^{12+}$ and $\mathrm{Ar}^{17+}$ has been analyzed in details using IS model within non relativistic as well as relativistic theory. We use this model 
Table 2. Relativistic and non-relativistic transition energy of $\mathrm{Al}^{12+}$ for different Debye screening parameter and box radius.

\begin{tabular}{|c|c|c|c|c|c|c|c|c|c|c|c|}
\hline \multirow[t]{2}{*}{ Ion } & \multirow[t]{2}{*}{$\begin{array}{l}\text { Plasma } \\
\text { density } \\
\text { (/c.c.) }\end{array}$} & \multirow[t]{2}{*}{$\begin{array}{l}\text { Temp. } \\
(\mathrm{eV})\end{array}$} & \multirow[t]{2}{*}{$\begin{array}{l}\text { Debye } \\
\text { para } \\
\text { (a.u.) }\end{array}$} & \multirow[t]{2}{*}{$\begin{array}{c}\text { Debye } \\
\text { Sh Rad } \\
\text { (a.u.) }\end{array}$} & \multicolumn{2}{|c|}{$\begin{array}{c}\text { Orbital } \\
\text { energy } \\
-E \text { (a.u.) }\end{array}$} & \multirow[t]{2}{*}{$\begin{array}{r}\text { Transition } \\
\text { scheme }\end{array}$} & \multicolumn{2}{|c|}{$\begin{array}{c}\text { Transition } \\
\text { energy } \\
\text { (a.u.) }\end{array}$} & \multicolumn{2}{|c|}{$\begin{array}{c}\text { Energy } \\
\text { shift } \\
(\mathrm{eV})\end{array}$} \\
\hline & & & & & Rel & Non-Rel & & Rel & Non-Rel & Rel & Non-Rel \\
\hline \multirow[t]{27}{*}{$\mathrm{Al}^{12+}$} & $1.0(22)$ & 300 & 0.154 & 6.50328 & 82.7066 & 82.5156 & $1 s \rightarrow 2 p$ & 63.49789 & 63.33468 & 1.0770 & 1.0972 \\
\hline & & & & & & & $3 p$ & 75.17195 & 74.99568 & 3.2009 & 3.1410 \\
\hline & & & & & & & $4 p$ & 79.17590 & 78.98747 & 6.1871 & 6.2935 \\
\hline & $1.5(22)$ & 300 & 0.188 & 5.30991 & 82.2731 & 82.0823 & $1 s \rightarrow 2 p$ & 63.47852 & 63.31529 & 1.6041 & 1.6248 \\
\hline & & & & & & & $3 p$ & 75.11382 & 74.95165 & 4.7827 & 4.3391 \\
\hline & & & & & & & $4 p$ & 79.07184 & 78.88048 & 9.0187 & 9.2048 \\
\hline & $2.0(22)$ & 300 & 0.217 & 4.59852 & 81.9048 & 81.7139 & $1 s \rightarrow 2 p$ & 63.45912 & 63.29586 & 2.1320 & 2.1535 \\
\hline & & & & & & & $3 p$ & 75.05604 & 74.90643 & 6.3550 & 5.5696 \\
\hline & & & & & & & $4 p$ & 78.97013 & 78.77558 & 11.7863 & 12.0593 \\
\hline & $2.5(22)$ & 300 & 0.243 & 4.11304 & 81.5756 & 81.3847 & $1 s \rightarrow 2 p$ & 63.43951 & 63.27621 & 2.6656 & 2.6882 \\
\hline & & & & & & & $3 p$ & 74.99892 & 74.85465 & 7.9093 & 6.9786 \\
\hline & & & & & & & $4 p$ & 78.86956 & 78.67419 & 14.5230 & 14.8182 \\
\hline & $3.0(22)$ & 300 & 0.266 & 3.75467 & 81.2852 & 81.0944 & $1 s \rightarrow 2 p$ & 63.42043 & 63.25709 & 3.1848 & 3.2085 \\
\hline & & & & & & & $3 p$ & 74.94271 & 74.79863 & 9.4388 & 8.5030 \\
\hline & & & & & & & $4 p$ & 78.77459 & 78.58558 & 17.1073 & 17.2294 \\
\hline & $3.5(22)$ & 300 & 0.288 & 3.47615 & 81.0081 & 80.8173 & $1 s \rightarrow 2 p$ & 63.40068 & 63.23729 & 3.7222 & 3.7473 \\
\hline & & & & & & & $3 p$ & 74.88485 & 74.73743 & 11.0133 & 10.1684 \\
\hline & & & & & & & $4 p$ & 78.67940 & 78.50974 & 19.6975 & 19.2932 \\
\hline & $4.0(22)$ & 300 & 0.308 & 3.25164 & 80.7568 & 80.5661 & $1 s \rightarrow 2 p$ & 63.38146 & 63.21803 & 4.2453 & 4.2714 \\
\hline & & & & & & & $3 p$ & 74.82883 & 74.67665 & 12.5377 & 11.8223 \\
\hline & & & & & & & $4 p$ & 78.58756 & 78.45786 & 22.1966 & 20.7049 \\
\hline & $4.5(22)$ & 300 & 0.326 & 3.06568 & 80.5311 & 80.3404 & $1 s \rightarrow 2 p$ & 63.36315 & 63.19967 & 4.7435 & 4.7710 \\
\hline & & & & & & & $3 p$ & 74.77574 & 74.61846 & 13.9823 & 13.4057 \\
\hline & & & & & & & $4 p$ & 78.51635 & 78.43443 & 24.1343 & 21.3424 \\
\hline & $5.0(22)$ & 300 & 0.344 & 2.90836 & 80.3059 & 80.1152 & $1 s \rightarrow 2 p$ & 63.34388 & 63.18036 & 5.2679 & 5.2964 \\
\hline & & & & & & & $3 p$ & 74.72022 & 74.55784 & 15.4931 & 15.0553 \\
\hline & & & & & & & $4 p$ & 78.45252 & 78.43180 & 25.8712 & 21.4140 \\
\hline
\end{tabular}

as it is simple and expected to produce results in reasonable agreement with the experimental data. The particular ions have been chosen here as laser produced plasma experiments in such systems exist [10-12] and spectral lines of Lyman series originating in plasma environments have been reported. Our aim is to see the reliability of the IS model of the plasma in predicting the experimentally observed lines of the Lyman series. The shifts can always be estimated from the free line positions. The orbital energies for different plasma coupling strengths have been obtained from the solution of the generalized eigenvalue equation (9) with respect to a limited basis set composed of linear combination of STO's. For $\mathrm{C}^{5+}$ ion we have chosen only a two parameter representation for the ground orbital and its reliability has been tested by comparing the eigen energy for the free systems. For $\mathrm{Al}^{12+}$ and $\mathrm{Ar}^{17+}$ we have chosen four parameter representation for the same. To study the excitation energies and transition wavelengths under plasma we used a twelve parameter representation of the first order perturbed orbitals for $\mathrm{C}^{5+}$ while an 8 parameters representation was adopted for $\mathrm{Al}^{12+}$ and $\mathrm{Ar}^{17+}$. For the case of $\mathrm{Al}^{12+}$ and $\mathrm{Ar}^{17+}$ the results for our detailed investigations using IS model with different electron densities have been displayed in Tables 1 and 3. We have considered the behavior of the ground state orbital energy and the transition energy to first three dipole allowed excited states $2 p, 3 p$ and $4 p$. The energy shifts have been calculated for $\mathrm{Al}^{12+}$ while for $\mathrm{Ar}^{17+}$, the wavelengths for the free as well as those in presence of plasma have been reported. This is because the data on the laser produced experiments on plasma for $\mathrm{Al}^{12+}[12]$ and $\mathrm{Ar}^{17+}$ [11] have been given accordingly. The other model which exists in literature is the so-called Debye model [4]. Here the effect due to the surrounding plasma is given by a screened Coulomb potential. In order to have an idea about how the results from Debye model compare with those from IS model as well as experimental data, we have computed the energy levels and other properties using Debye model with spherical confinement, the radius of confinement being the inverse of the Debye screening parameter which determines the sphere of influence. Here the effective potential is given by [4]

$$
V_{e f f}(r)=-\frac{Z e^{-\mu r}}{r}
$$

where $Z$ is the nuclear charge and $\mu$ is the Debye screening parameter given by

$$
\mu=\left[\frac{4 \pi(1+Z) n}{\kappa T}\right]^{\frac{1}{2}}
$$


Table 3. Relativistic and non-relativistic transition energy of $\mathrm{Ar}^{17+}$ for different Ion-Sphere (IS) radius.

\begin{tabular}{|c|c|c|c|c|c|c|c|c|c|}
\hline \multirow[t]{2}{*}{ Ion } & \multirow{2}{*}{$\begin{array}{c}\text { Plasma } \\
\text { density } \\
\text { (/c.c.) }\end{array}$} & \multirow{2}{*}{$\begin{array}{c}\text { IS } \\
\text { radius } \\
\text { (a.u.) } \\
\end{array}$} & \multicolumn{2}{|c|}{$\begin{array}{c}\text { Orbital } \\
\text { energy }-E \text { (a.u.) }\end{array}$} & \multirow[t]{2}{*}{$\begin{array}{r}\text { Transition } \\
\text { scheme }\end{array}$} & \multicolumn{2}{|c|}{$\begin{array}{c}\text { Transition } \\
\text { energy (a.u.) }\end{array}$} & \multicolumn{2}{|c|}{$\begin{array}{c}\text { Transition } \\
\text { wave length }(\AA)\end{array}$} \\
\hline & & & Rel & Non-Rel & & Rel & Non-Rel & Rel & Non-Rel \\
\hline \multirow[t]{48}{*}{$\operatorname{Ar}^{17+}$} & \multirow[t]{3}{*}{$9.54(20)$} & \multirow[t]{3}{*}{30.0} & \multirow[t]{3}{*}{161.8549} & \multirow[t]{3}{*}{161.1500} & $1 s \rightarrow 2 p$ & 122.10220 & 121.49997 & 3.7305 & 3.7490 \\
\hline & & & & & $3 p$ & 144.66130 & 143.99982 & 3.1488 & 3.1633 \\
\hline & & & & & $4 p$ & 152.56180 & 151.87442 & 2.9857 & 2.9992 \\
\hline & \multirow[t]{3}{*}{$2.58(22)$} & \multirow[t]{3}{*}{10.0} & \multirow[t]{3}{*}{160.1549} & \multirow[t]{3}{*}{159.4500} & $1 s \rightarrow 2 p$ & 122.10153 & 121.49929 & 3.7306 & 3.7491 \\
\hline & & & & & $3 p$ & 144.65685 & 143.99535 & 3.1489 & 3.1633 \\
\hline & & & & & $4 p$ & 152.54681 & 151.86477 & 2.9860 & 2.9994 \\
\hline & \multirow[t]{3}{*}{$1.0(23)$} & \multirow[t]{3}{*}{6.4941} & \multirow[t]{3}{*}{158.7785} & 158.0736 & $1 s \rightarrow 2 p$ & 122.09966 & 121.49741 & 3.7306 & 3.7491 \\
\hline & & & & & $3 p$ & 144.64459 & 143.98306 & 3.1492 & 3.1636 \\
\hline & & & & & $4 p$ & 152.50553 & 151.81798 & 2.9868 & 3.0004 \\
\hline & $2.0(23)$ & 5.1543 & 157.7581 & 157.0533 & $1 s \rightarrow 2 p$ & 122.09709 & 121.49482 & 3.7307 & 3.7492 \\
\hline & & & & & $3 p$ & 144.62769 & 143.96644 & 3.1495 & 3.1640 \\
\hline & & & & & $4 p$ & 152.44872 & 151.75973 & 2.9879 & 3.0015 \\
\hline & $3.0(23)$ & 4.5027 & 157.0425 & 156.3376 & $1 s \rightarrow 2 p$ & 122.09452 & 121.49223 & 3.7308 & 3.7493 \\
\hline & & & & & $3 p$ & 144.61075 & 143.95059 & 3.1499 & 3.1643 \\
\hline & & & & & $4 p$ & 152.39194 & 151.70093 & 2.9891 & 3.0027 \\
\hline & $4.0(23)$ & 4.0190 & 156.3612 & 155.7680 & $1 s \rightarrow 2 p$ & 122.09138 & 121.48964 & 3.7309 & 3.7494 \\
\hline & & & & & $3 p$ & 144.59007 & 143.93565 & 3.1503 & 3.1647 \\
\hline & & & & & $4 p$ & 152.32277 & 151.62842 & 2.9904 & 3.0041 \\
\hline & $5.0(23)$ & 3.7978 & 155.9918 & 155.2869 & $1 s \rightarrow 2 p$ & 122.08937 & 121.48705 & 3.7309 & 3.7494 \\
\hline & & & & & $3 p$ & 144.57679 & 143.92126 & 3.1506 & 3.1650 \\
\hline & & & & & $4 p$ & 152.27847 & 151.58148 & 2.9913 & 3.0050 \\
\hline & $6.0(23)$ & 3.5738 & 155.5713 & 154.8665 & $1 s \rightarrow 2 p$ & 122.08680 & 121.48447 & 3.7310 & 3.7495 \\
\hline & & & & & $3 p$ & 144.55975 & 143.90649 & 3.1510 & 3.1653 \\
\hline & & & & & $4 p$ & 152.22179 & 151.52097 & 2.9924 & 3.0062 \\
\hline & $7.0(23)$ & 3.3948 & 155.1954 & 154.4906 & $1 s \rightarrow 2 p$ & 122.08422 & 121.48187 & 3.7311 & 3.7496 \\
\hline & & & & & $3 p$ & 144.54269 & 143.89068 & 3.1514 & 3.1657 \\
\hline & & & & & $4 p$ & 152.16513 & 151.46022 & 2.9935 & 3.0074 \\
\hline & $8.0(23)$ & 3.2470 & 154.8538 & 154.1490 & $1 s \rightarrow 2 p$ & 122.08165 & 121.47928 & 3.7312 & 3.7497 \\
\hline & & & & & $3 p$ & 144.52559 & 143.87384 & 3.1517 & 3.1660 \\
\hline & & & & & $4 p$ & 152.10852 & 151.39962 & 2.9946 & 3.0086 \\
\hline & $9.0(23)$ & 3.1220 & 154.5396 & 153.8348 & $1 s \rightarrow 2 p$ & 122.07908 & 121.47669 & 3.7313 & 3.7498 \\
\hline & & & & & $3 p$ & 144.50846 & 143.85638 & 3.1521 & 3.1664 \\
\hline & & & & & $4 p$ & 152.05193 & 151.33965 & 2.9957 & 3.0098 \\
\hline & $1.0(24)$ & 3.0143 & 154.2480 & 153.5431 & $1 s \rightarrow 2 p$ & 122.07650 & 121.47410 & 3.7313 & 3.7498 \\
\hline & & & & & $3 p$ & 144.49129 & 143.83861 & 3.1525 & 3.1668 \\
\hline & & & & & $4 p$ & 151.99539 & 151.28082 & 2.9969 & 3.0110 \\
\hline & $1.5(24)$ & 2.6332 & 153.0251 & 152.3203 & $1 s \rightarrow 2 p$ & 122.06361 & 121.46113 & 3.7317 & 3.7502 \\
\hline & & & & & $3 p$ & 144.40493 & 143.74921 & 3.1544 & 3.1688 \\
\hline & & & & & $4 p$ & 151.71395 & 151.01987 & 3.0024 & 3.0162 \\
\hline & $1.8(24)$ & 2.4780 & 152.4192 & 151.7144 & $1 s \rightarrow 2 p$ & 122.05588 & 121.45334 & 3.7320 & 3.7505 \\
\hline & & & & & $3 p$ & 144.35266 & 143.69573 & 3.1555 & 3.1699 \\
\hline & & & & & $4 p$ & 151.54573 & 150.90012 & 3.0057 & 3.0186 \\
\hline & $2.0(24)$ & 2.3924 & 152.0519 & 151.3471 & $1 s \rightarrow 2 p$ & 122.05071 & 121.44814 & 3.7321 & 3.7506 \\
\hline & & & & & $3 p$ & 144.31763 & 143.66004 & 3.1563 & 3.1707 \\
\hline & & & & & $4 p$ & 151.43565 & 150.83746 & 3.0079 & 3.0199 \\
\hline & $2.5(24)$ & 2.2209 & 151.2303 & 150.5256 & $1 s \rightarrow 2 p$ & 122.03780 & 121.43514 & 3.7325 & 3.7510 \\
\hline & & & & & $3 p$ & 144.22930 & 143.57026 & 3.1582 & 3.1727 \\
\hline & & & & & $4 p$ & 151.15811 & 150.73922 & 3.0135 & 3.0218 \\
\hline
\end{tabular}

$\mu$ is a function of the temperature $T$ and number density $n$ of the plasma electrons. One can simulate a large number of plasma conditions by properly choosing $n$ and $T$. Using the potential function given by equation (13) with a given parameter $\mu$, one can proceed in the same way as is being done in the strongly coupled plasma model to study the behavior of orbital energies and excitation properties.
In such calculations we have chosen the plasma temperature $T$ as reported in the experimental papers $[11,12]$ and varies the electron density $n$ to get the screening parameters $\mu$. For each $\mu$ value we have chosen the radius of confinement as $R=\frac{1}{\mu}$ which effectively gives the Debye sphere of influence. The spatial confinement with respect to the Debye radius is incorporated in the numerical 
Table 4. Relativistic and non-relativistic transition energy of $\mathrm{Ar}^{17+}$ for different Debye screening parameter and box radius.

\begin{tabular}{|c|c|c|c|c|c|c|c|c|c|c|c|}
\hline \multirow[t]{2}{*}{ Ion } & \multirow[t]{2}{*}{$\begin{array}{c}\text { Plasma } \\
\text { density } \\
\text { (/c.c.) }\end{array}$} & \multirow[t]{2}{*}{$\begin{array}{c}\text { Temp } \\
(\mathrm{eV})\end{array}$} & \multirow[t]{2}{*}{$\begin{array}{c}\text { Debye } \\
\text { para } \\
\text { (a.u.) }\end{array}$} & \multirow[t]{2}{*}{$\begin{array}{c}\text { Debye } \\
\text { radius } \\
\text { (a.u.) }\end{array}$} & \multicolumn{2}{|c|}{$\begin{array}{c}\text { Orbital } \\
\text { energy }-E \\
\text { (a.u.) }\end{array}$} & \multirow[t]{2}{*}{$\begin{array}{r}\text { Tran. } \\
\text { sch. }\end{array}$} & \multicolumn{2}{|c|}{$\begin{array}{c}\text { Transition } \\
\text { energy } \\
\text { (a.u.) }\end{array}$} & \multicolumn{2}{|c|}{$\begin{array}{c}\text { Transition } \\
\text { wave length } \\
(\AA)\end{array}$} \\
\hline & & & & & Rel & Nol-Rel & & Rel & Nol-Rel & Rel & Nol-Rel \\
\hline \multirow[t]{9}{*}{$\operatorname{Ar}^{17+}$} & $\overline{1.0(23)}$ & 1000 & 0.3103 & 3.2230 & 157.1904 & 156.4865 & $1 s \rightarrow 2 p$ & 121.94107 & 121.33840 & 3.7355 & 3.7540 \\
\hline & & & & & & & $3 p$ & 144.17601 & 143.52411 & 3.1594 & 3.1737 \\
\hline & & & & & & & $4 p$ & 151.67941 & & 3.0031 & \\
\hline & $5.0(23)$ & 1000 & 0.6938 & 1.4414 & 150.5664 & 149.8640 & $1 s \rightarrow 2 p$ & 121.33281 & 120.72769 & 3.7542 & 3.7730 \\
\hline & & & & & & & $3 p$ & 142.49773 & 141.51795 & 3.1966 & 3.2187 \\
\hline & & & & & & & $4 p$ & 150.25318 & & 3.0316 & \\
\hline & $1.0(24)$ & 1000 & 0.9812 & 1.0192 & 145.7360 & 145.0367 & $1 s \rightarrow 2 p$ & 120.61125 & 119.98983 & 3.7767 & 3.7962 \\
\hline & & & & & & & $3 p$ & 141.17721 & 138.52859 & 3.2265 & 3.2882 \\
\hline & $5.0(24)$ & 1000 & 2.1939 & 0.4558 & 126.5337 & 125.8566 & $1 s \rightarrow 2 p$ & 116.71007 & 111.28980 & 3.9029 & 4.0930 \\
\hline
\end{tabular}

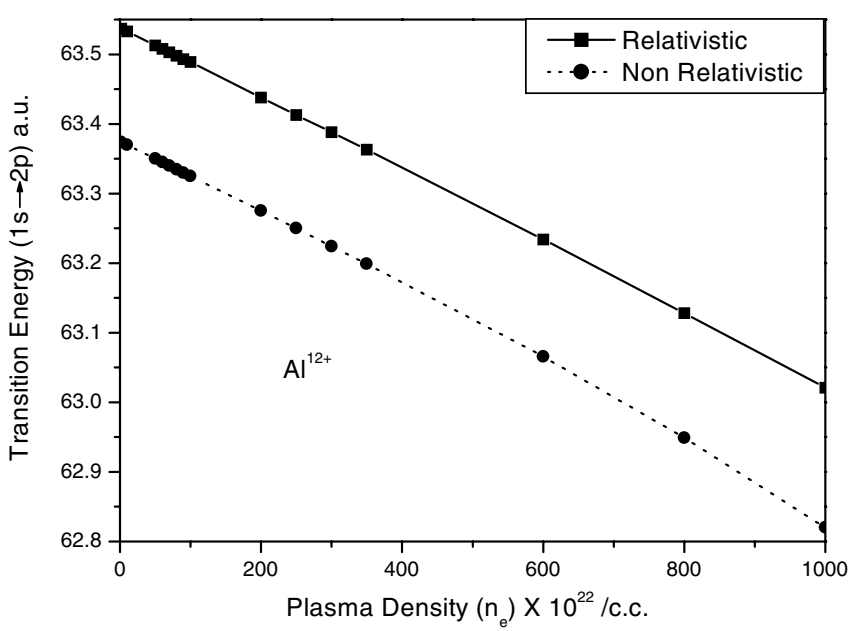

Fig. 1. Plot of the relativistic and non relativistic transition energy $(1 s \rightarrow 2 p)$ (a.u.) obtained by using IS model against plasma electron density (/cc) for $\mathrm{Al}^{12+}$.

calculations in exactly the same way as is being done for the Ion Sphere (IS) model. Such results have been displayed in Tables 2 and 4 for the respective cases of $\mathrm{Al}^{12+}$ and $\mathrm{Ar}^{17+}$. The number of parameters for the ground and excited state functions are identical in the Debye plasma and in the IS models. In Tables 1 to 4 the transition energies from the $1 s \rightarrow 2 p, 3 p$ and $4 p$ states have been reported for the cases only in which the excited state is bound. As soon as the transition energy exceeds that of the ionization energy for increased plasma strength, it goes in the continuum and such cases have not been displayed in the tables. Experimental shift for the Lyman $\alpha\left(\mathrm{Ly}_{\alpha}\right)$ line for $\mathrm{Al}^{12+}$ with estimated electron density $n \sim(5-10) \times 10^{23} /$ cc and temperature $T \sim 300 \mathrm{eV}$ is given by $3.7 \pm 0.7 \mathrm{eV}$ [12]. Our calculation using IS model at $n=2.5 \times 10^{24} /$ cc yields a value $3.41 \mathrm{eV}$ whereas a quantum mechanical calculations of Nguyen et al. [27] based on collision theory yields a value $3.5 \mathrm{eV}$ at $n=8 \times 10^{23} / \mathrm{cc}$ and $T \sim 300 \mathrm{eV}$.

Figure 1 shows the general trend of the transition energy $1 s \rightarrow 2 p$ for $\mathrm{Al}^{12+}$ against the Ion Sphere radius $R$ with non relativistic and relativistic models. For the rel-

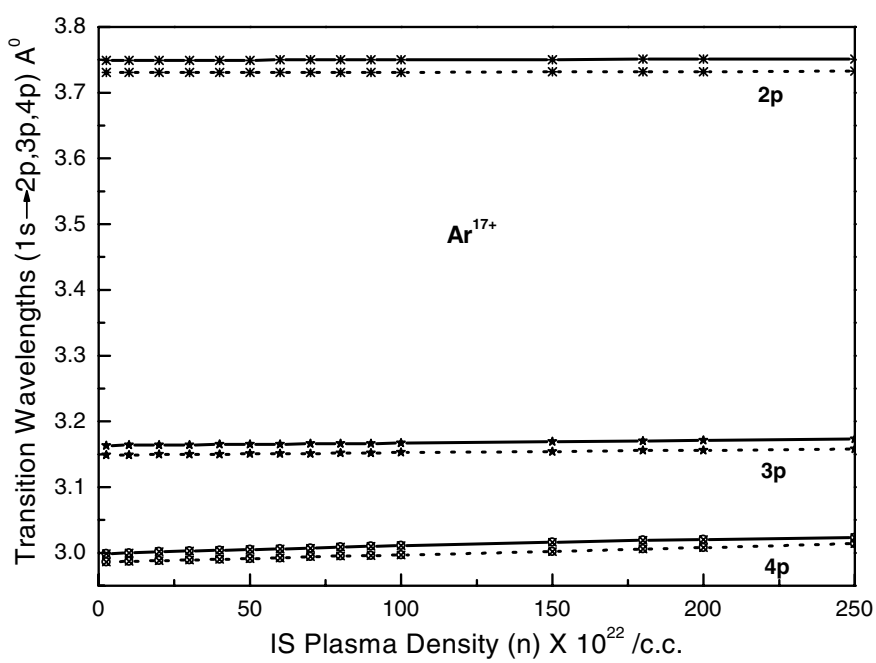

Fig. 2. Plot of the relativistic (dotted line with symbols) as well as non relativistic transition (solid line with symbols) wavelength $(1 s \rightarrow 2 p, 3 p, 4 p)(\AA)$ obtained by using IS model against plasma electron density (/cc) for $\mathrm{Ar}^{17+}$.

ativistic case weighted average of the $p_{\frac{3}{2}}$ and $p_{\frac{1}{2}}$ state energies have been reported all throughout. It appears that the relativistic results differ only at higher plasma electron densities. In Figure 2 we plotted the non relativistic and relativistic transition wavelengths $1 s \rightarrow 2 p$, $3 p$ and $4 p$ against IS plasma density for $\mathrm{Ar}^{17+}$. The relativistic effects are little more pronounced here as the nuclear charge $Z$ is larger. Figure 3 displays a comparison of our calculated results for the transition wavelengths for $\mathrm{Ar}^{17+}$ using non relativistic as well as relativistic methods within Ion Sphere (IS) model and spatially confined Debye screening model with the laser plasma experimental data. The wavelengths for the $1 s \rightarrow 2 p, 3 p, 4 p$ transitions as obtained from an analysis of the experimental data [11] are $3.7494,3.2394$ and $2.9927 \AA$ respectively. They are in reasonable agreement with the calculated theoretical results using IS model with electron density $10^{24} / \mathrm{cc}$. The laser plasma experiment by Nantel et al. [10] yields data on hydrogen and helium like spectra of $\mathrm{C}$ under strong 

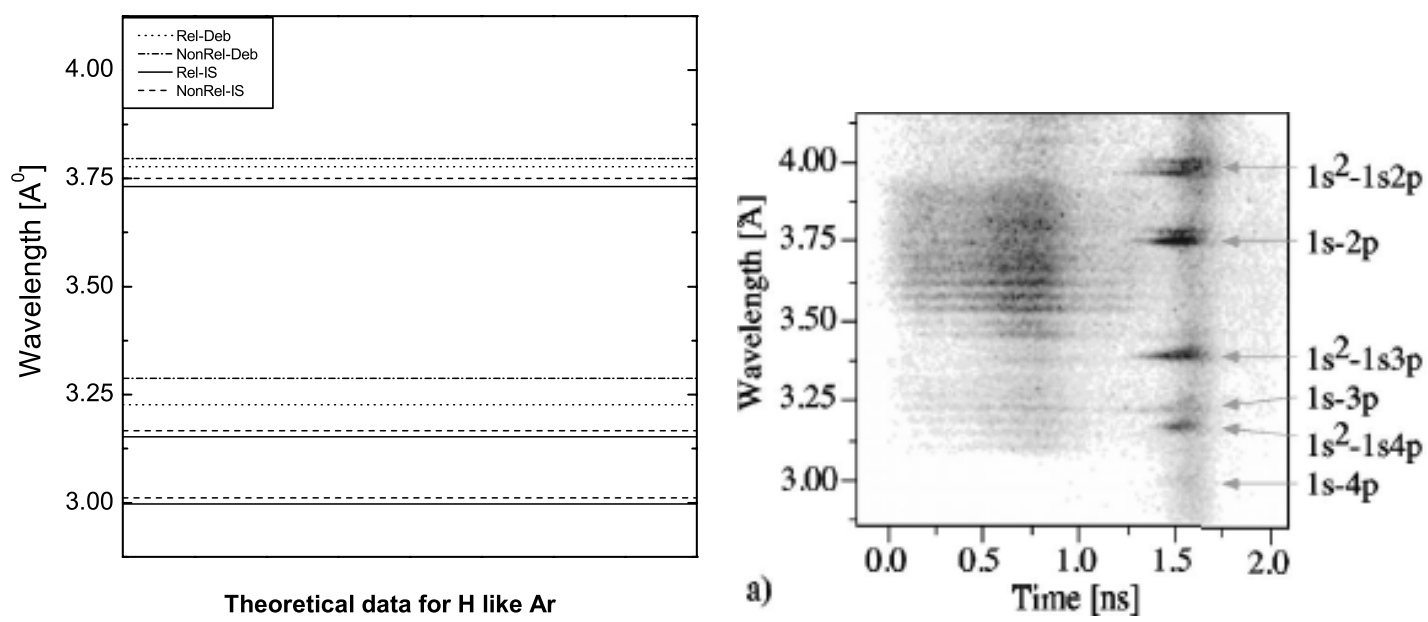

Fig. 3. Comparison between the experimental results and that obtained theoretically by using Ion Sphere as well as Debye plasma model for $1 s \rightarrow 2 p, 3 p, 4 p$ transition wavelength $(\AA)$ of $\operatorname{Ar}^{17+}$. We have taken same plasma temperature $(T=1000 \mathrm{eV})$ and density $\left(n_{e}=10^{24}\right.$ c.c.) as was in the experiment. The experimental figure has been taken from reference [11].

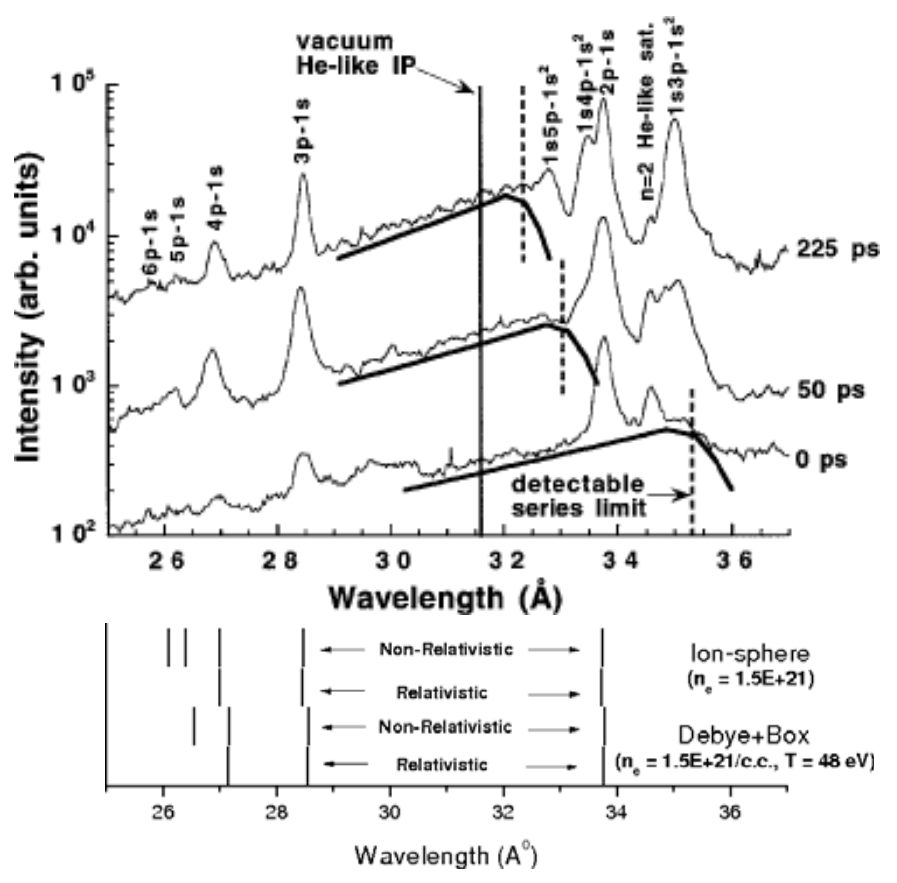

Fig. 4. Comparison between the experimental results $\left(\mathrm{C}^{4+}\right.$ and $\mathrm{C}^{5+}$ ) and that obtained theoretically by using Ion Sphere as well as Debye plasma model for $1 s \rightarrow 2 p, 3 p, 4 p, 5 p, 6 p$ transition wavelength $(\AA)$ of hydrogen like carbon. The experimental figure has been taken from reference [10].

plasma with estimated density of $n=1.5 \times 10^{21} / \mathrm{cc}$ and temperature $48 \mathrm{eV}$. We have performed non relativistic and relativistic estimates of the positions of Lyman lines of $\mathrm{C}^{5+}$ using the Ion Sphere (IS) model at experimental density and spatially confined Debye plasma model at the same density and temperature. In Figure 4 we displayed our results along with those obtained by Nantel et al. [10]. We observed very reasonable fitting with the experimental lines positions for the Lyman transitions $1 s \rightarrow 2 p, 3 p, 4 p$, $5 p$ and $6 p$ for which the respective wavelengths as obtained from an analysis of the line profiles are 33.7491, 28.4667, 26.8791, 26.2104 and 25.7380 in $\AA$. It appears that with IS model non relativistic and relativistic estimates at $n=1.5 \times 10^{21} / \mathrm{cc}$ and temperature $48 \mathrm{eV}$ agree well with experimental data, while there are little variations with confined Debye plasma model. This is reflected in Table 5, where we compare the experimental data with our theoretical estimates for $\mathrm{C}^{5+}$ and $\mathrm{Ar}^{17+}$ using non relativistic as well relativistic models. Since we are concerned with hydrogenic spectra in which no electron correlation effect is present, our theoretical results should be accurate enough to be compared with experimental data. The accuracy of our results could be assessed from a comparison with the energy levels for the free system for which we get exact agreement. The accuracy of the experimental data is difficult to assess as most of the authors do not furnish the error bars. The experiments deal with complex situation with plasma in non LTE where density and temperature evolve. The experimental condition assumes a simulated temperature and density which has a range. We have performed the calculations with the reported density and temperature and tried to obtain the best fit with the experiments.

\section{Conclusion}

From the analysis of the calculated data by using IS and Debye models one can conclude that IS model, though simple, yields very reasonable theoretical estimates of spectral line positions and shifts of the spectral lines obtained from laser produced plasmas. It can be a viable method for the understanding of the experimental observations on strongly coupled plasmas obtained in laboratory and astrophysics. 
Table 5. Comparison between experimental and theoretical results of relativistic and non-relativistic transition wavelength of $\mathrm{C}^{5+}$ and $\mathrm{Ar}^{17+}$. The experimental data have been extracted from Figure 1 of reference [10] and Figure 2a of reference [11] for $\mathrm{C}^{5+}$ and $\mathrm{Ar}^{17+}$ respectively.

\begin{tabular}{|c|c|c|c|c|c|}
\hline \multirow[t]{3}{*}{ Excitation } & \multicolumn{5}{|c|}{ Transition wavelength $(\AA)$} \\
\hline & \multicolumn{2}{|c|}{ IS model results } & \multicolumn{2}{|c|}{ Debye model results } & \multirow[t]{2}{*}{ Experimental results } \\
\hline & Non-relativistic & Relativistic & Non-relativistic & Relativistic & \\
\hline \multicolumn{6}{|c|}{$\mathrm{C}^{5+}$} \\
\hline $1 s \rightarrow 2 p$ & 33.7422 & 33.7238 & 33.7878 & 33.7693 & 33.7491 \\
\hline $1 s \rightarrow 3 p$ & 28.4733 & 28.4589 & 28.5693 & 28.5546 & 28.4667 \\
\hline $1 s \rightarrow 4 p$ & 27.0055 & 26.9918 & 27.1597 & 27.1427 & 26.8791 \\
\hline $1 s \rightarrow 5 p$ & 26.3906 & & 26.5488 & & 26.2104 \\
\hline $1 s \rightarrow 6 p$ & 26.0926 & & 25.9557 & & 25.7380 \\
\hline \multicolumn{6}{|c|}{$\operatorname{Ar}^{17+}$} \\
\hline $1 s \rightarrow 2 p$ & 3.7498 & 3.7311 & 3.7962 & 3.7767 & 3.7494 \\
\hline $1 s \rightarrow 3 p$ & 3.1668 & 3.1525 & 3.2882 & 3.2265 & 3.2394 \\
\hline $1 s \rightarrow 4 p$ & 3.0110 & 2.9969 & & & 2.9927 \\
\hline
\end{tabular}

The authors (PKM and SF) are thankful to AvH foundation for financial assistance towards mutual visits of Indian and German scientists. The financial assistance from the Department of Science and Technology (DST), Govt. of India under research grant no. SR/S2/LOP-05/2005 is gratefully acknowledged.

\section{References}

1. B. Tabbert, H. Günther, G. zu Putlitz, J. Low. Temp. Phys. 109, 653 (1997)

2. W. Jaskolski, Phys. Rep. 271, 1 (1996)

3. J.P. Connerade, V.K. Dolmatov, P.A. Lakshmi, J. Phys. B, At. Mol. Opt. Phys. 33, 251 (2000)

4. A.I. Akhiezer, I.A. Akhiezer, R.A. Polovin, A.G. Sitenko, K.N. Stepanov, Plasma Electrodynamics, linear response theory (Pergamon, Oxford, 1975), Vol. 1

5. S. Ichimaru, Rev. Mod. Phys. 54, 1017 (1982)

6. B.A. Hammel, C.J. Keane, M.D. Cable, D.R. Kania, J.D. Kilkenny, R.W. Lee, R. Pasha, Phys. Rev. Lett. 70, 1263 (1993)

7. L. DaSilva, A. Ng, B.K. Godwal, G. Chiu, F. Cottet, M.C. Richardson, P.A. Jaanimagi, Phys. Rev. Lett. 62, 1623 (1989); A. Djaoui et al., Plasma Phys. Contr. Fus. 31, 111 (1989)

8. E. Leboucher-Dalimier, A. Poquerusse, P. Angelo, I. Gharbi, H. Derfoul, J. Quant. Spectrosc. Radiat. Transfer 51, 187 (1994)

9. J. Workman, M. Nantel, A. Maksimchuk, D. Umstadter, Appl. Phys. Lett. 70, 312 (1997)

10. M. Nantel, G. Ma, S. Gu, C.Y. Cote, J. Itatani, D. Umstadter, Phys. Rev. Lett. 80, 4442 (1998)

11. N.C. Woolsey, B.A. Hammel, C.J. Keane, C.A. Back, J.C. Moreno, J.K. Nash, A. Calisti, C. Mosse, R. Stamm, B.
Talin, A. Asfaw, L.S. Klein, R.W. Lee, Phys. Rev. E 57, 4650 (1998)

12. A. Saemann, K. Eidmann, I.E. Golovkin, R.C. Mancini, E. Andersson, E. Forster, K. Witte, Phys. Rev. Lett. 82, 4843 (1999)

13. J.C. Stewart Jr, K.D. Pyatt, Astrophys. J. 144, 1203 (1966)

14. B.F. Rozsnyai, Phys. Rev. A 43, 3035 (1991)

15. D. Ray, Phys. Rev. E 62, 4126 (2000)

16. Y.D. Jung, Eur. Phys. J. D 7, 249 (1999), and references therein

17. H.R. Griem, Phys. Rev. A 15, 2943 (1988); H.R. Griem Spectral Line Broadening by Plasmas (Academic Press, New York, 1974)

18. J. Seidel, S. Arndt, W.D. Kraeft, Phys. Rev. E 52, 5387 (1995)

19. S. Skupski, Phys. Rev. A 21, 1316 (1980)

20. U. Gupta, A.K. Rajagopal, Phys. Rept. 87, 259 (1982)

21. B. Saha, P.K. Mukherjee, G.H.F. Diercksen, Astron. Astrophys. 396, 337 (2002)

22. A.N. Sil, B. Saha, P.K. Mukherjee, Int. J. Quantum Chem. 104, 903 (2005)

23. B. Saha, P.K. Mukherjee, D. Bielinska-Waz, Jacek Karwowski, J. Quant. Spectrosc. Radiat. Transfer 92, 1, (2005); 78, 131 (2003)

24. A.N. Sil, P.K. Mukherjee, Int. J. Quantum Chem. 106, 465 (2006)

25. S. Fritzsche, J. Electr. Spec. Rel. Phenom. 114-116, 1155 (2001); S. Fritzsche, Phys. Scr. T100, 37 (2002)

26. P.O. Löwdin, P.K. Mukherjee, Chem. Phys. Lett. 14, 1 (1972)

27. H. Nguyen, M. Koenig, D. Benredjem, M. Caby, G. Coulaud, Phys. Rev. A 33, 1279 (1986) 